\title{
Matching game based resource allocation algorithm for energy-harvesting small cells network with NOMA
}

\author{
Xueting Wang ${ }^{1,2}$, Qi Zhu ${ }^{1,2^{*}}$ \\ 1 Key Wireless Laboratory of Jiangsu Province, School of Telecommunication and Information Engineering, \\ Nanjing University of Posts and Telecommunications, Nanjing 210003, China. \\ 2 Key Lab on Wideband Wireless Communications and Sensor Network Technology of Ministry of Education, \\ Nanjing University of Posts and Telecommunications, Nanjing, Jiangsu 210003, China \\ [e-mail:1051473941@qq.com; zhuqi@njupt.edu.cn] \\ *Corresponding author: Qi Zhu
}

Received April 9, 2018; revised May 11, 2018; accepted June 3, 2018;

published November 30, 2018

\begin{abstract}
In order to increase the capacity and improve the spectrum efficiency of wireless communication systems, this paper proposes a rate-based two-sided many-to-one matching game algorithm for energy-harvesting small cells with non-orthogonal multiple access (NOMA) in heterogeneous cellular networks (HCN). First, we use a heuristic clustering based channel allocation algorithm to assign channels to small cells and manage the interference. Then, aiming at addressing the user access problem, this issue is modeled as a many-to-one matching game with the rate as its utility. Finally, considering externality in the matching game, we propose an algorithm that involves swap-matchings to find the optimal matching and to prove its stability. Simulation results show that this algorithm outperforms the comparing algorithm in efficiency and rate, in addition to improving the spectrum efficiency.
\end{abstract}

Keywords: energy harvest; heterogeneous networks; matching game; NOMA

This research has been supported by The National Natural Science Foundation of China(NO.61571234, NO.61631020), Postgraduate Research \& Practice Innovation Program of Jiangsu Province(NO.KYCX17_0765). 


\section{Introduction}

The explosive growth of mobile devices and the rapidly increase of wideband wireless services call for advanced communication techniques that can achieve high spectral efficiency and meet massive connectivity requirement[1]. Considering as an important system of 5G, the ultra dense network (UDN) is an effective technology for enhancing the capacity of wireless networks. Facing the significant challenges of spectrum shortage and mobile equipment proliferation, effective methods should be involved in the UDN. Owing to its advantages in improving spectrum efficiency and energy efficiency and realizing the benefits of flexible traffic offload [2][3], NOMA is widely studied in combination with wireless communication networks to increase network capacity. Energy harvesting is a technology that harvests renewable energy, such as solar and wind resources, from the environment, which is key technology in 5G networks[4]. By introducing this technology in small cell networks, green telecommunication can be realized and small cells can be deployed even when a power grid is not available. With a proper resource allocation algorithm, small cell networks can have better capacity and service performance. Hence, this paper combines NOMA, UDN, and energy-harvesting small cells, then proposes a novel resource allocation algorithm to improve the system performance.

\section{Related Work}

More and more studies on NOMA and energy harvesting using matching game have emerged, as it is an attractive research topic related to small cells. In [5], a wireless backhaul power allocation algorithm considering the practical network energy consumption is given in multitier small cell networks. In [6], a study on the fair-NOMA algorithm is presented, mainly focusing on the power allocation problem. In [7], the authors analytically characterized the optimal power allocation with given channel assignments over multiple channels under different performance criteria in a NOMA system. In [8], a study of the non-orthogonal spectrum assignment with the goal of maximizing the social welfare of the network is discussed. This work adopts the many-to-one stable matching game framework to address this problem. In [9], a joint optimization of spectrum allocation and power control is presented with the aim of maximizing the users' sum rate and realization of fairness. Cognitive radios are considered in [10], and the channel allocation problem of primary users with secondary users is modeled as a one-to-one matching. In [11], a study of the resource allocation and user scheduling problem for a downlink non-orthogonal multiple access network is presented, which aims to jointly optimize the sub-channel assignment and the power allocation to maximize the weighted total sum-rate while taking into account of user fairness.

However, all the researches mentioned above did not address the energy-harvesting opportunities in a small cell, which is considered promising for 5G. When energy harvesting is involved, the power allocation of small cells is expected to become more complex, leading to more challenges in the optimization of power. In addition, the power control algorithm discussed in [5] considers one center base station instead of multiple pieces of equipment, which is not practical. In the approaches discussed in [6], [7], [8], [9], and [11] the access problem is not taken into consideration. Only in [10] a matching algorithm is used to solve the user access problem by one-to-one matching game. However, this algorithm provides no 
contribution to solving multi-user access problem in small cell networks with NOMA. Many-to-one matching game can match several players of one type with one player of another type, which is suitable for our model. The matching game is a kind of cooperative game, which can enhance the players' utility by cooperation, which is contrary to the concept of non-cooperative games. Thus, in this paper, we study a many-to-one matching game algorithm for users accessing energy-harvesting small cells with NOMA in heterogeneous cellular networks.

To solve the resource allocation problem, we propose a two-sided many-to-one matching game algorithm with the rate as its utility. First, a heuristic clustering based channel allocation algorithm is used to mitigate interference. Then, we model the user access problem as a matching game with users and small cells as players, whose utilities are data rates. With a view to improving the spectrum efficiency, NOMA and successive interference cancellation (SIC) are also introduced. In addition, the externality exists in the matching game for involving NOMA. Therefore, we propose an algorithm to solve this problem and find the optimal matching in a given network. Finally, the proof of our algorithm's stability is also stated to show its optimality. In contrast to the existing work on NOMA, this paper mainly focuses on the multiuser access problem, and models it as a many-to-one matching game. By designing an algorithm to find the global optimization results and reallocating the power in small cells, the performance of small cell networks is further improved. The main contributions of this paper are as follows:

(1)We establish a system model that employs NOMA and energy-harvesting in small cells of a HCN. In this model, the energy arrives in the small cells with a uniform distribution that has upper and lower bounds. Considering NOMA and energy-harvesting, the power allocation in small cells is expected to meet the requirement of NOMA, and the restrain of harvesting energy. In addition, the co-tier interference and cross-tier interference in HCN are included, and the issue is mitigated by channel allocation.

(2)We model the multiuser access problem as a many-to-one matching game. In contrast to the previously published works, we assume that the small cells in this scenario serve multiple users simultaneously, and use a many-to-one matching game to address the user access problem. In order to acquire the optimal matching results, we also provide a swap-matchings algorithm to find the global optimization, and prove its stability.

(3)To acquire better capacity and to realize NOMA, we also present a novel power allocation method in small cells. According to [12], the signal should satisfy the requirement of NOMA, or it can not be decoded successfully. With the aim on improving system capacity, we propose a new power allocation algorithm to provide users with better channel conditions and greater power under the power constraints of NOMA.

\section{System model and algorithm}

\subsection{System model}

As shown in Fig. 1, the downlink data transmission scenario of a two-tier HCN with underlay model is considered, where the energy-harvesting small cell network and the macrocell network are deployed in the same area. We assume there are $N$ small cells and $K$ users in the scenario, and denote sets of small cells and users as $\{S B S\}=\left\{S B S_{1}, S B S_{2}, \ldots, S B S_{N}\right\}$ and $\{U E\}=\left\{U E_{1}, U E_{2}, \ldots, U E_{K}\right\}$ respectively. 


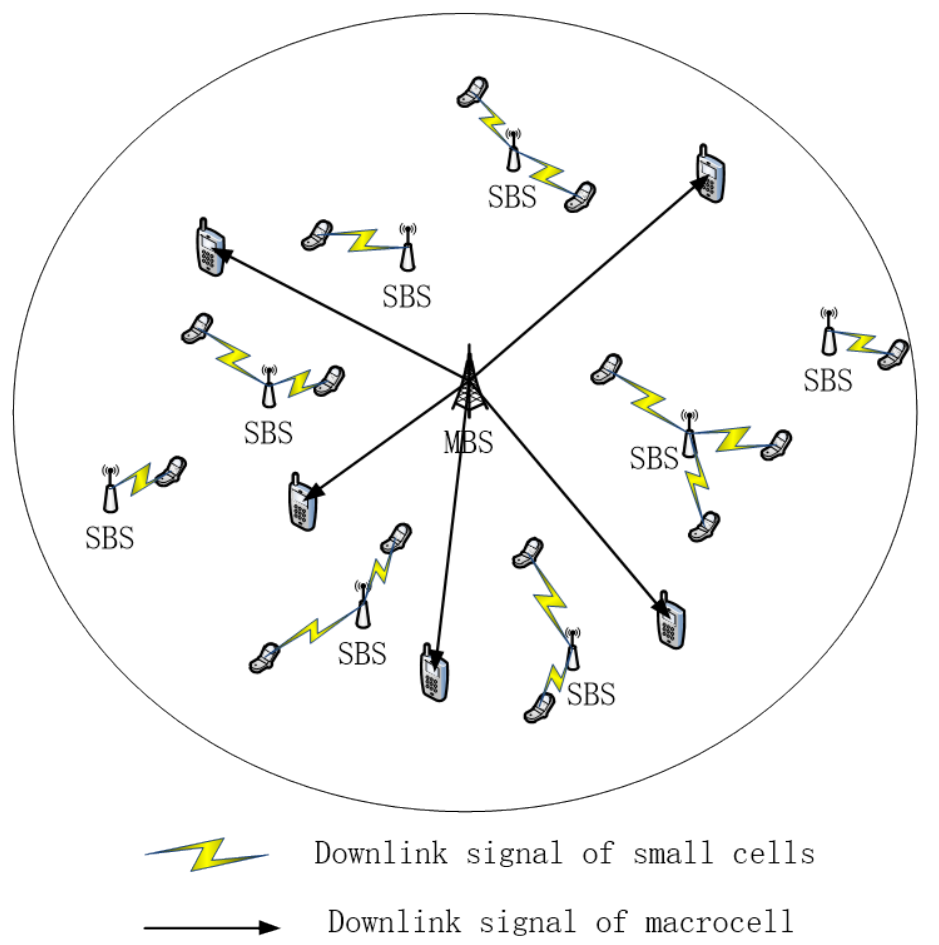

Fig. 1. Downlink scenario in two-tier network

In this system, the set of channels is $\{1,2, \ldots, I\}$ whose number is $I$. To mitigate the interference, a small cell occupies only one channel, and multiple users can access the same small cell simultaneously with NOMA. We assume that the user set in small cell $\boldsymbol{n}$ is denoted as $\left\{C L U_{n}\right\}$, and we sort the users in $\left\{C L U_{n}\right\}$ in descending order of channel gain between them and small cells. Then, we obtain the user access cluster $\left\{C L U_{n}\right\}_{n \in N}$. As most papers define, we define the arrival of energy is at the beginning of each time slot, and it is consumed within this time slot instead of storage. Under this definition, the harvesting energy set of small cells in the beginning of a time slot is $\{E\}=\left\{E_{1}, E_{2}, \ldots, E_{N}\right\}$, and the transmission power set is $\left\{P_{n}^{s}\right\}_{n \in N}=\left\{\frac{E_{n}}{T}\right\}_{n \in N}$, where $T$ is the duration of the time slot.

In order to analyse the interference involved by NOMA in a small cell, we assume, without loss of generality, $U E_{k}$ and $U E_{f}$ are served at the same time with NOMA in $S B S_{n}$ and $G_{n, k}^{S, U}>G_{n, f}^{S, U}$, where $G_{n, k}^{S, U}$ and $G_{n, f}^{S, U}$ represent channel gains from $S B S_{n}$ to $U E_{k}$ and $U E_{f}$. In order to retrieve $U E_{k}$ 's signal by SIC, $U E_{f}$ 's signal should be decoded successfully first and removed from the overlapped signal, which means the transmission power from $S B S_{n}$ to $U E_{f}$ should be greater than that to $U E_{k}$, i.e. $P_{n, k}^{S}<P_{n, f}^{S}$. Thus, for $U E_{f}$, it will suffer interference from the signal $S B S_{n}$ sends to $U E_{k}$ and this interference can't be canceled by SIC[13]. 
In this scenario, for the number of channels is less than number of small cells, small cells need to reuse channels with other small cells and macrocell, resulting in the interference among them. To improve the efficiency of spectrum, NOMA is used to serve multiple users in the same small cell with the same channel, which also introduces NOMA interference among users in the same channel of a small cell. Ranking users in small cells in descending order of $G_{n, k}^{S, U}$, we obtain user access set $\left\{C L U_{n}\right\}_{n \in N}$. The interference and noise suffered by $U E_{k}$ in $S B S_{n}$ on channel $i \in\{1,2, \ldots, I\}$ can be expressed as

$$
\operatorname{Igan}_{k}^{n, i}=\sum_{j \in N / n} c_{i, j} * P_{j}^{S} * G_{j, k}^{S, U}+P^{M} * G_{k}^{M, U}+\sum_{G_{n, f}^{S, U}>G_{n, k}^{S, U}, f \in\left\{C L U_{n}\right\}} P_{n, f}^{S} * G_{f}^{S, U}+N 0 * B,
$$

Where $G_{j, k}^{S, U}$ denotes the channel gain between $S B S_{j}$ and $U E_{k}$, and $c_{i, j} \in\{0,1\}$ indicates whether $S B S_{j}$ uses the channel $i$. In other word, $c_{i, j}=1$ means $S B S_{j}$ uses channel $i$, and $c_{i, j}=0$ means not. $P_{j}^{S}$ and $P^{M}$ represent transmission power of $S B S_{j}$ and the macrocell in each channel respectively. Hence, the co-tier interference and the cross-tier interference are expressed as $\sum_{j \in N / n} c_{i, j} * P_{j}^{S *} G_{j, k}^{S, U}$ and $P^{M} * G_{k}^{M, U}$, in which $G_{k}^{M, U}$ is the channel gain between macro base station and $U E_{k}$. The interference in same small cells causing by NOMA should be included as well, which is formulated as $\sum_{G_{n, f}^{S, U}>G_{n, k}^{S, U}, f \in\left\{C L U_{n}\right\}} P_{n, f}^{S} * G_{f}^{S, U}$. Besides the interference, the background noise should not be forgotten and is denoted as $N 0 * B$, where $B$ is the bandwidth of channel. The signal to interference and noise ratio (SINR) of $U E_{k}$ on channel $i$ can be formulated as

$$
\gamma_{k}^{n, i}=\frac{P_{n, k}^{S} * G_{n, k}^{S, U}}{\sum_{j \in N / n} c_{i, j} * P_{j}^{S} * G_{j, k}^{S, U}+\sum_{G_{n, f}^{S, U}>G_{n, k}^{S, U}, f \in\left\{C L U_{n}\right\}} P_{n, f}^{S} * G_{f}^{S, U}+P^{M} * G_{k}^{M, U}+N 0 * B} .
$$

The data rate is expressed as follow

$$
\begin{aligned}
R_{k}^{n, i} & =B * \log _{2}\left(1+\gamma_{k}^{n, i}\right) \\
& =B * \log _{2}\left(1+\frac{P_{n, k}^{S} * G_{n, k}^{S, U}}{\sum_{j \in N / n} c_{i, j} * P_{j}^{S} * G_{j, k}^{S, U}+\sum_{G_{n, f}^{S, U}>G_{n, k}^{S, U}, f \in\left\{C L U_{n}\right\}} P_{n, f}^{S} * G_{f}^{S, U}+P^{M} * G_{k}^{M, U}+N 0 * B}\right) .
\end{aligned}
$$

\subsection{A heuristic clustering based channel allocation algorithm}

In order to mitigate the interference causing by channel-sharing, a heuristic clustering based channel allocation algorithm[14] can be carried out first.

In this algorithm, a weighted directional graph $G=(V, E, W)$ of the small cell network is established firstly, where the small cell set is $\{V\}=\left\{v_{1}, v_{2}, \ldots, v_{N}\right\}$ and $\{E\}=\left\{e_{n, m}\right\}_{n, m \in N}$, $e_{n, m} \in\{0,1\}$ denotes the set of interference among small cells. If $e_{n, m}=1$, it means that $S B S_{m}$ will cause interference to $S B S_{n}$, while $e_{n, m}=0$ means there is no interference between them. In order to evaluate the interference among small cells, we use $\{W\}=\left\{w_{n, m}\right\}_{n, m \in N}$ to represent the set of weight on each line. We can define the 
interference from $S B S_{m}$ to $S B S_{n}$, as $w_{n, m}$, which can be formulated as [14]

$$
w_{n, m}=\max \left(G_{n, m}^{S, S}-\delta, 0\right),
$$

Where $\delta$ is the receiving sensitivity of small cells and $G_{n, m}^{S, S}$ is denoted as the channel gain from $S B S_{m}$ to $S B S_{n}$. (4) requires that the channel gain should be greater than the receiving sensitivity, or the interference could be ignored.

Based on the algorithm, the set of small cells should be divided into $I$ clusters, where $I$ is the number of channels, and the set of clusters is expressed as $\left\{C_{h}\right\}_{h \in\{I\}}$. Let $W_{h}=\sum_{n, m \in C_{h}} w_{n, m}$ denote the sum of weight in cluster $C_{h}$ and $w_{n}=\sum_{m=1, n \neq m}^{N} w_{n, m}$ denote the sum weight of $S B S_{n}$ from other small cells. Assuming $S B S_{m}$ attending cluster $C_{h}$, the increased weight in cluster can be formulated as $W_{n}^{h}=\sum_{m \in C_{n}} w_{m, n}$. By choosing the cluster satisfying the less increased weight requirement for each user and allocating a channel for each cluster, the interference could be mitigated effectively. The process of this channel allocation algorithm is as follow:

(1) each small cell calculates $w_{n}$ and then we obtain the small cell set $\left\{V^{\prime}\right\}$ by sorting $w_{n}$ in descending order;

(2) choose the top $I$ small cells from $\left\{V^{\prime}\right\}$ and assign them to $I$ clusters separately, then delete these small cells from $\left\{V^{\prime}\right\}$;

(3) get a small cell from $\left\{V^{\prime}\right\}$ in order and choose a cluster $C_{h}$ with minimum $w_{h}$ to attend;

(4) after clustering, assign a channel to each cluster and the algorithm is over.

\subsection{User access algorithm using matching game theory}

Considering the shortcoming of non-cooperative game and improving the efficiency of spectrum, we formulate the user access problem as a two-sided many-to-one matching game, which is one of cooperative games, to match multi users with a small cell. The quota of small cells is $a$, which means a small cell could serve $a$ users at most. The quota of users is only one, which represents a user can only access one small cell. With the target of this algorithm being to maximize the sum rate of users, formally, we define the user access optimization problem as

$$
\begin{array}{ll}
\max & \sum_{i \in I} \sum_{n \in N} \sum_{k \in K} A_{k}^{n, i} * R_{k}^{n, i} \\
\text { s.t. } & \sum_{i \in I} \sum_{k \in K} A_{k}^{n, i} * P_{n, k}^{S}=P_{n}^{S}, \\
& 0 \leq \sum_{i \in I} \sum_{k \in K} A_{k}^{n, i} \leq a, A_{k}^{n, i} \in\{0,1\}, \forall n \in N .
\end{array}
$$

Where $A_{k}^{n, i}$ denotes whether the $U E_{k}$ accesses $S B S_{n}$ which uses the channel $i$. This optimization problem aims at maximizing the sum rate of all users with the limitation of harvested energy and quota of users a small cell can serve. To realize this goal, we solve this 
problem with many-to-one user access matching game other than one-to-one matching game [10][15] and define the two-sided game as below:

The matching between users in $K$ and energy-harvesting small cells in $N$ can be formulated as $\left(K, N, \succ_{K}, \succ_{N}\right)$, in which $\succ_{K}=\left\{\succ_{k}\right\}_{k \in K}$ and $\succ_{N}=\left\{\succ_{n}\right\}_{n \in N}$ are on behalf of the preference of users and small cells on the other player. For $U E_{k}$, if it prefers $S B S_{n}$ than $S B S_{m}$, it can be formulated as $n \succ_{k} m$. Similarly, $S B S_{n}$ prefers $U E_{k}$ than $U E_{k^{\prime}}$ can be defined as $k \succ_{n} k^{\prime}$.

Definition 1: A matching game $\mu$ is defined as a function from the set of $K \bigcup N$ into the set of $K \bigcup N$, where $k$ and $n$ satisfy $k \in K$ and $n \in N$ :

(1) $\mu(k) \in N,|\mu(k)|=1$;

(2) $\mu(n) \subset K, 0 \leq|\mu(n)| \leq a$;

(3) $k \in \mu(n)$ if and only if $\mu(k)=n$;

Due to allow multi users to access one small cell with the only channel via NOMA, interference among these users should be considered when allocating the transmission power for them. Assuming the users in $S B S_{n}$ is $\left\{C L U_{n}\right\}$ with its number as $\left|\left\{C L U_{n}\right\}\right|$ and $G_{n, f}^{S, U}>G_{n, k}^{S, U}, f, k \in\left\{C L U_{n}\right\}$ represents the users whose channel gain are larger than that of than $U E_{k}$ in $\left\{C L U_{n}\right\}$. Thus, the interference from these users to $U E_{k}$ is formulated as $\sum_{G_{n, f}^{S, U}>G_{n,,}^{S, U}, f \in\left\{C L U_{n \in N}\right\}} P_{n, f}^{S} * G_{f}^{S, U}$. In order to decode information from overlapped signal successfully by SIC, the SINR threshold must be met for each signal on each receiver. As we known in [12], users with NOMA suffer interference from other NOMA users of same small cells. While using SIC[13], a user can decode interference signals of NOMA users which have greater transmission power than its one by one and then remove them from the overlapping signal it receives. Finally, the signal of this user is obtained. But to decode each interference signal and its signal successfully, these signals should satisfy the requirement of SINR. We assume all receivers have the same SINR threshold requirement and denote it as $S I N R_{t h}$. By defining the power of $S B S_{n}$ is divided into $\left|\left\{C L U_{n}\right\}\right|$ parts and the set is $\left\{P_{1}^{S}, P_{2}^{S}, \ldots, P_{\left|\left\{C L U_{n}\right\}\right|}^{S}\right\}$, this requirement[12] can be defined as follow

$$
\frac{P_{2}^{S}}{P_{1}^{S}} \geq \operatorname{SINR}_{t h}, \frac{P_{3}^{S}}{P_{1}^{S}+P_{2}^{S}} \geq \operatorname{SINR}_{t h}, \ldots, \frac{P_{\left\{C L U_{n}\right\} \mid}^{S\left[C L U_{n}\right\} \mid-1}}{\sum_{i=1}^{S} P_{i}^{S}} \geq \operatorname{SINR}_{t h} .
$$

In (6), $P_{1}^{S}$ is the interference of $P_{2}^{S}, P_{1}^{S}$ and $P_{2}^{S}$ are the interference of $P_{3}^{S}$. The ratio of the signal and power must no less than $S I N R_{t h}$. Or signals can not be decoded.

The users in $\left\{C L U_{n}\right\}$ have already been sorted by channel gain from $S B S_{n}$ in descending order. Aiming at maximizing throughput of system, it is obviously beneficial to assign more power to the user with larger channel gain. Thus the " $\geq$ " in (6) could be changed to "=", and we can obtain the transmission power of all users in $S B S_{n}$ : 
(1) IF the number of access users is greater than 1 , then

$$
P_{n, C L U_{n}(j)}^{S}=\left\{\begin{array}{lc}
P_{n}^{S} /\left(\operatorname{SINR}_{t h}\right)^{\left|\left\{C L U_{n}\right\}\right|} & j=1 ; \\
\frac{P_{n}^{S} * \operatorname{SINR}_{t h} *\left(\operatorname{SINR}_{t h}\right)^{j-2}}{\left(S I N R_{t h}\right)^{\left|\left\{C L U_{n}\right\}\right|}} & 2 \leq j \leq a ;
\end{array}\right.
$$

$j$ is the sequence number in $\left\{C L U_{n}\right\}$ after sorting and $C L U_{n}(j)$ represents the $j$-th user in $\left\{C L U_{n}\right\}$.

(2) IF the number of access user is 1 , then

$$
P_{n, k}^{S}=P_{n}^{S} .
$$

In (8), $U E_{k}$ is the only user in $\left\{C L U_{n}\right\}$.

After determining the transmission power, the rate of users can be obtained via (2) and (3). We define the utility of users and small cells to be the achievable rate, thus the utility function of user is written as below

$$
U_{K}(k, n)=R_{k}^{n, i}
$$

And the small cell's utility is

$$
U_{N}(k, n)=R_{k}^{n, i}
$$

From (6), (7) and (8), we can know that the transmission power and preference lists will vary with the change of user-small cell associations, which is called externality[16], and the externality will influence the stable matching. Considering this problem and the concept of swap-matching in [17][18], we give a definition of stable matching, which is also the optimal matching result:

Definition 2: (1) for a given pair of small cells $m, n \in N$, a given pair of users $k, k^{\prime} \in K$ and user access sets $\left\{C L U_{m}\right\},\left\{C L U_{n}\right\}$, a matching $\mu$ with matching pairs $\left(k^{\prime}, m\right),(k, n) \quad$ is stable if no stable swap-matchings $\mu_{n, m}^{k, k^{\prime}}=\left\{\mu /\left\{\left(k^{\prime}, m\right),(k, n)\right\}\right\} \bigcup\left\{(k, m)\left(k^{\prime}, n\right)\right\} \quad\left(\quad\left\{C L U_{m}\right\}_{n, m}^{k, k^{\prime}}=\left\{C L U_{m}\right\} / k^{\prime} \bigcup k \quad\right.$, $\left.\left\{C L U_{n}\right\}_{n, m}^{k, k^{\prime}}=\left\{C L U_{n}\right\} / k \cup k^{\prime}\right)$ exist and satisfy $\mu_{n, m}^{k, k^{\prime}}=\left\{\mu /\left\{\left(k^{\prime}, m\right),(k, n)\right\}\right\} \bigcup\left\{(k, m)\left(k^{\prime}, n\right)\right\}$, $U_{x}\left(\mu_{n, m}^{k, k^{\prime}}\right) \geq U_{x}(\mu)$ and $\exists x \in\left\{m, n,\left\{C L U_{m}\right\}_{n, m}^{k, k^{\prime}},\left\{C L U_{n}\right\}_{n, m}^{k, k^{\prime}}\right\}, U_{x}\left(\mu_{n, m}^{k, k^{\prime}}\right)>U_{x}(\mu)$;

(2) for a given matching pair $(k, n)$, small cell $m$ and user access sets $\left\{C L U_{m}\right\},\left\{C L U_{n}\right\}$, a matching $\mu$ is stable if no stable swap-matchings $\mu_{n, m}^{k}=\{\mu /(k, n)\} \bigcup(k, m) \quad\left(\left\{C L U_{m}\right\}_{n, m}^{k}=\left\{C L U_{m}\right\} \bigcup k,\left\{C L U_{n}\right\}_{n, m}^{k}=\left\{C L U_{n}\right\} / k\right)$ exist and satisfy $\forall x \in\left\{m, n,\left\{C L U_{m}\right\}_{n, m}^{k},\left\{C L U_{n}\right\}_{n, m}^{k}\right\} \quad, \quad U_{x}\left(\mu_{n, m}^{k}\right) \geq U_{x}(\mu) \quad$ and $\exists x \in\left\{m, n,\left\{C L U_{m}\right\}_{n, m}^{k},\left\{C L U_{n}\right\}_{n, m}^{k}\right\}, U_{x}\left(\mu_{n, m}^{k}\right)>U_{x}(\mu)$.

If such two situations don't exist in final matching $\mu$, the matching $\mu$ is a stable matching. Aiming at obtaining the stable matching, we propose an externality-considered two-sided user access algorithm in this section. First, each user choose the nearest small cell to access to form the initial matching $\mu^{0}$. Then based on $\mu^{0}$, users try to access another small cell or exchange association with other users. Once the swap action improves the utility as described in definition 2, user will sent an application to the small cell. After receiving all applications, each small cell decides which application to accept and reject 
others. With finite iterations, a stable matching can be achieved eventually. In conclusion, the details of this algorithm are shown in Table 1.

Table 1. Two-sided many-to-one user access matching algorithm

Algorithm 1: Two-sided many-to-one user access matching algorithm

1. initializing: user set is $\{U E\}=\left\{U E_{1}, U E_{2}, \ldots, U E_{K}\right\}$, energy-harvesting small cell set is $\{S B S\}=\left\{S B S_{1}, S B S_{2}, \ldots, S B S_{N}\right\}, t=1, \mu^{0}=0$, small cell quota is $a$;

2. For $k=1: K$

3. User $k$ sorts the small cells on distance from small cells in descending order, and send an application to the nearest small cell $n$; if the small cell is full, user $k$ will

try to access the next small cell in turn until it is accepted; a match pair $(k, n) \quad$ is formed;

4. End

5. According to the match pairs in step 3, the initial matching $\mu^{0}$ is formed. Using (7) (10), users and small cells calculate current utility $U_{x}\left(\mu^{0}\right)$;

6. While $\mu^{t}=\mu^{t-1} \| t=1$

7. $\mu^{t}=\mu^{t-1}$;

8. For $k=1: K$

9. Assume exchange sets $\{E X 1\}=\{0\},\{E X 2\}=\{0\}$ and utility sets $\{U 1\}=\{0\}$, $\{U 2\}=\{0\}$

10.

$$
\text { For } k^{\prime}=1: K
$$

11. Calculate the utility of $x \in\left\{m, n,\left\{C L U_{m}\right\}_{n, m}^{k, k^{\prime}},\left\{C L U_{n}\right\}_{n, m}^{k, k^{\prime}}\right\}$ and compare them with $U_{x}\left(\mu^{t-1}\right)$. if the exchange meets the requirements

12. in definition 2, $\{U 1\}=\{U 1\} \bigcup U_{x}\left(\mu_{n, m}^{k, k^{\prime}}\right), \quad\{E X 1\}=\{E X 1\} \bigcup\left(k, k^{\prime}\right)$; 13. 14.

For $m=1: N$

Calculate the utility of $x \in\left\{m, n,\left\{C L U_{m}\right\}_{n, m}^{k},\left\{C L U_{n}\right\}_{n, m}^{k}\right\}$ and compare them with $U_{x}\left(\mu^{t-1}\right)$. if the exchange meets the requirements in definition $2,\{U 2\}=\{U 2\} \cup U_{x}\left(\mu_{n, m}^{k}\right)$,

15. $\{E X 2\}=\{E X 2\} \bigcup(k, m)$; If $\{E X 1\} \neq \varnothing \|\{E X 2\} \neq \varnothing$

17. Choose the exchange pair in $\{U 1\}$ and $\{U 2\}$ that improve system most;

18.

If the exchange pair in $\{E X 1\}$ is selected update $\mu^{t}=\mu_{n, m}^{k, k^{\prime}}$;

20.

Else if exchange pair in $\{E X 2\}$ is selected

21. update $\mu^{t}=\mu_{n, m}^{k}$; 
22. $\quad$ End
23. End
24. End
25. $t=t+1$;
26. End

To ensure our algorithm having a stable matching result, we shall also take the stability into consideration and prove it.

Proposition 1: According to the definition 2, a swap takes place only when utilities of the involved players are strictly increased. With a given scenario, the number of small cells and that of users are fixed, that indicates an optimal matching must exist and only a finite number of swaps will occur in the current matching until it becomes optimal. As described in Table 1, our algorithm will not terminate until there is no swap-matching in the current matching. Therefore, the matching with our algorithm will converge to the optimal matching gradually. Hence, we can draw a conclusion that the swap-matching that can further improve the utility doesn't exist in the matching result of our algorithm, that is to say, the matching is stable and optimal.

\section{Simulation and result analysis}

In this section, simulation results on performance of our algorithm and another one are evaluated by matlab. We compare algorithm of [10] with our algorithm and consider a simulation scenario of one macro cell with radius of $200 \mathrm{~m}$. In our simulation, users and small cells are deployed with uniform distribution within macrocell's coverage. As described in [19], the energy harvesting process is characterized by the energy supply rate $\eta_{n}(t)$, which determines the amount of harvested energy of sensor $n$ in time slot $t$. Furthermore, $\eta_{n}(t)$ randomly varies in an i.i.d fashion over slots. The harvested energy $e_{n}(t)$ is bounded by $\eta_{n}(t)$, i.e.,

$$
0 \leq e_{n}(t) \leq \eta_{n}(t), \forall n \in N .
$$

This paper also assumes that the energy supply rate $\eta_{n}(t)$ is a uniform distribution in $\left[0, \eta_{\max }\right]$. Based on the definition in [19], we apply this model and assume the harvested energy of small cell $E_{n}$ in time slots is uniformly distributed in [ $E_{\min }, E_{\max }$, where $E_{\text {min }}$ and $E_{\max }$ are respectively the upper bound and lower bound. We assume the energy arrives in small cells with uniform distribution in [0.3 0.4]J. Channel model is cited in [20] and the formulas of channel gain are given as follow.

(1) If $d \leq 15$, which means the distance $d$ between transmitter and receiver is smaller than 15 meters, the channel gain is

$$
G=-(40+25 * \lg (d)+10) d B .
$$

(2) If $d>15$, which means the distance $d$ between transmitter and receiver is larger than 15 meters, the channel gain is:

$$
G=-(60+25 * \lg (15)+40 * \lg (d-15)+10) d B .
$$


Table 2. Simulation parameters

\begin{tabular}{|c|c|}
\hline Simulation parameters & Value \\
\hline Channel bandwidth $B$ & $500 \mathrm{kHZ}$ \\
\hline Number of users $K$ & {$[1038]$} \\
\hline Number of small cells $N$ & {$[610]$} \\
\hline Number of channels $I$ & 3 \\
\hline Range of harvesting energy & {$[0.30 .4] \mathrm{J} / \mathrm{s}$} \\
\hline Coverage of a macro cell & $200 \mathrm{~m}$ \\
\hline Quota of a small cell $a$ & 3 \\
\hline Transmission power of a macrocell per channel & $2 \mathrm{~W}$ \\
\hline
\end{tabular}

A comparison of the two algorithms on the users' average rate while varying the user number and small cell number is shown in Fig. 2. From this figure, it can be concluded that the average rate of users gradually increases with the increase of small cells. However, at one point, the rate decreases as more users are added. The reason being, with more users or less small cells, the average users in a small cell increases, which means that the transmission power as well as the rate of each user is decreased. In addition, our algorithm has a better performance than comparing one. This is because this algorithm is an optimal matching algorithm considering the externality while the one-to-one matching in comparing algorithms is optimal but do not consider the externality, which obviously increases the rate in a given system.

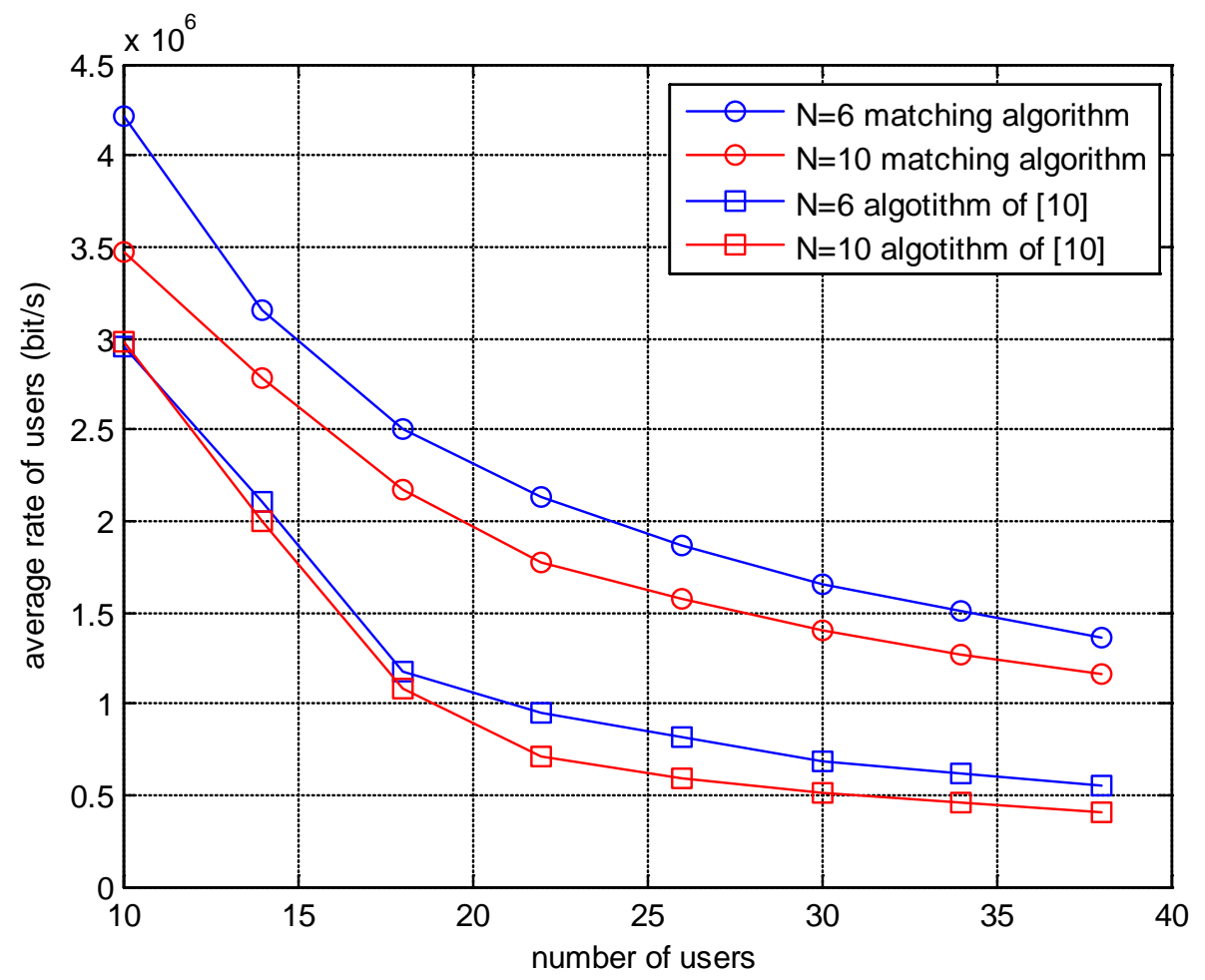

Fig. 2. Comparison on average rate of users 
Fig. 3 illustrates the variation of small cell energy efficiency. We can see that the energy efficiency of our algorithm is greater than that of comparing algorithm, for our algorithm serves multiple users simultaneously in a small cell and finds the optimal matching. In contrast, the comparing algorithm divides the users into two groups and establishes a one-to-one matching, which is not optimal and definitely less efficient. Besides, to our knowledge, the more power it uses, the less energy-efficient it becomes. Thus, when the number of users increases or small cell decreases, it leads to more users in a small cell and less transmission power for users, which definitely causes the increasement of energy efficiency.

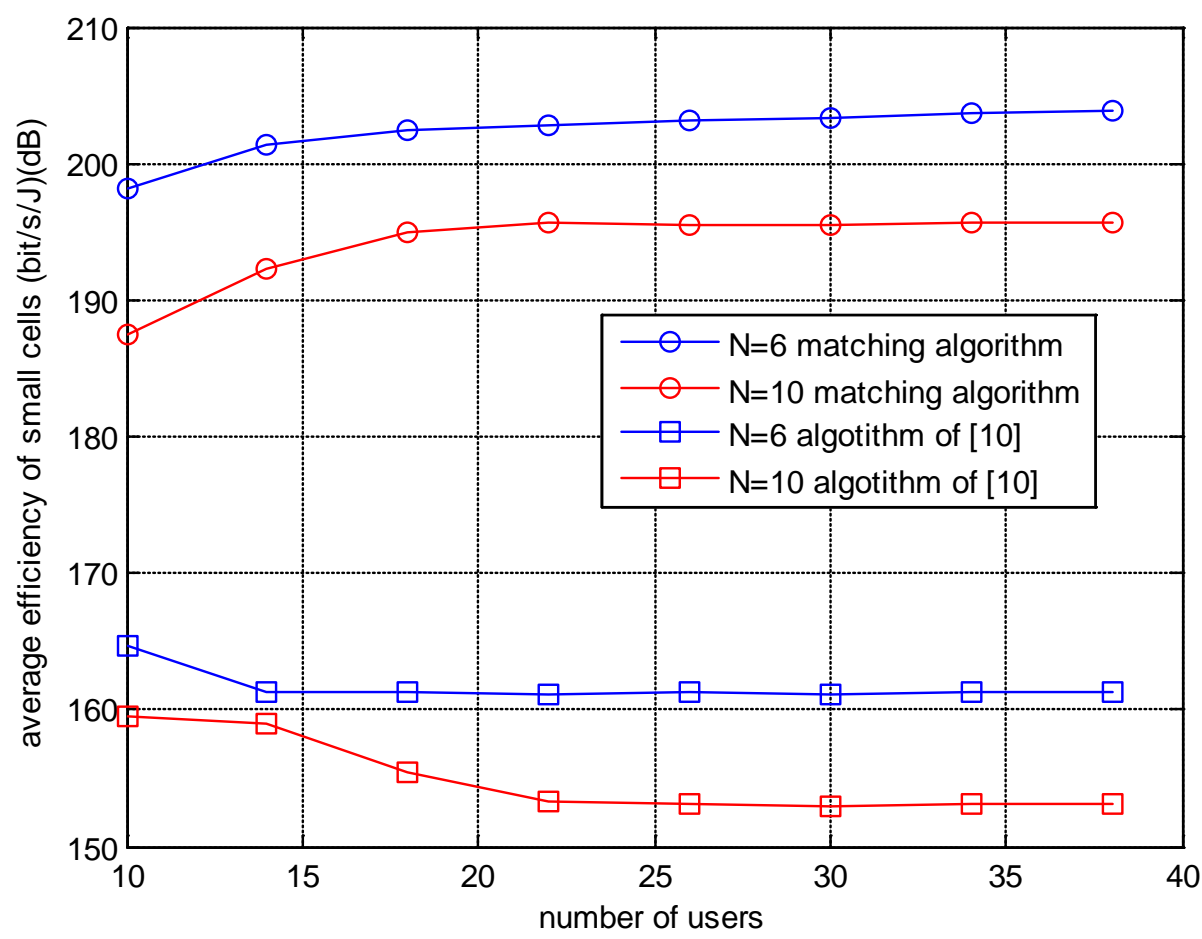

Fig. 3. Comparison on average energy efficiency of small cells

In Fig. 4, we compare the average user access ratio of the two algorithms, and the conclusion can be drawn that our algorithm has a higher access ratio. This is because our algorithm considers multiple users can access a small cell while the comparing one only allows at most two users in a small cell. In addition, the access ratio becomes greater when the number of small cells increases and the number of users decreases. The reason is that, with more small cells, the small cell network can serve more users when the number of users is given, resulting in the increase of the access ratio. But when the number of small cells is given, the number of users that can access the small cells is limited, and if more users are added, the ratio is decreased. 


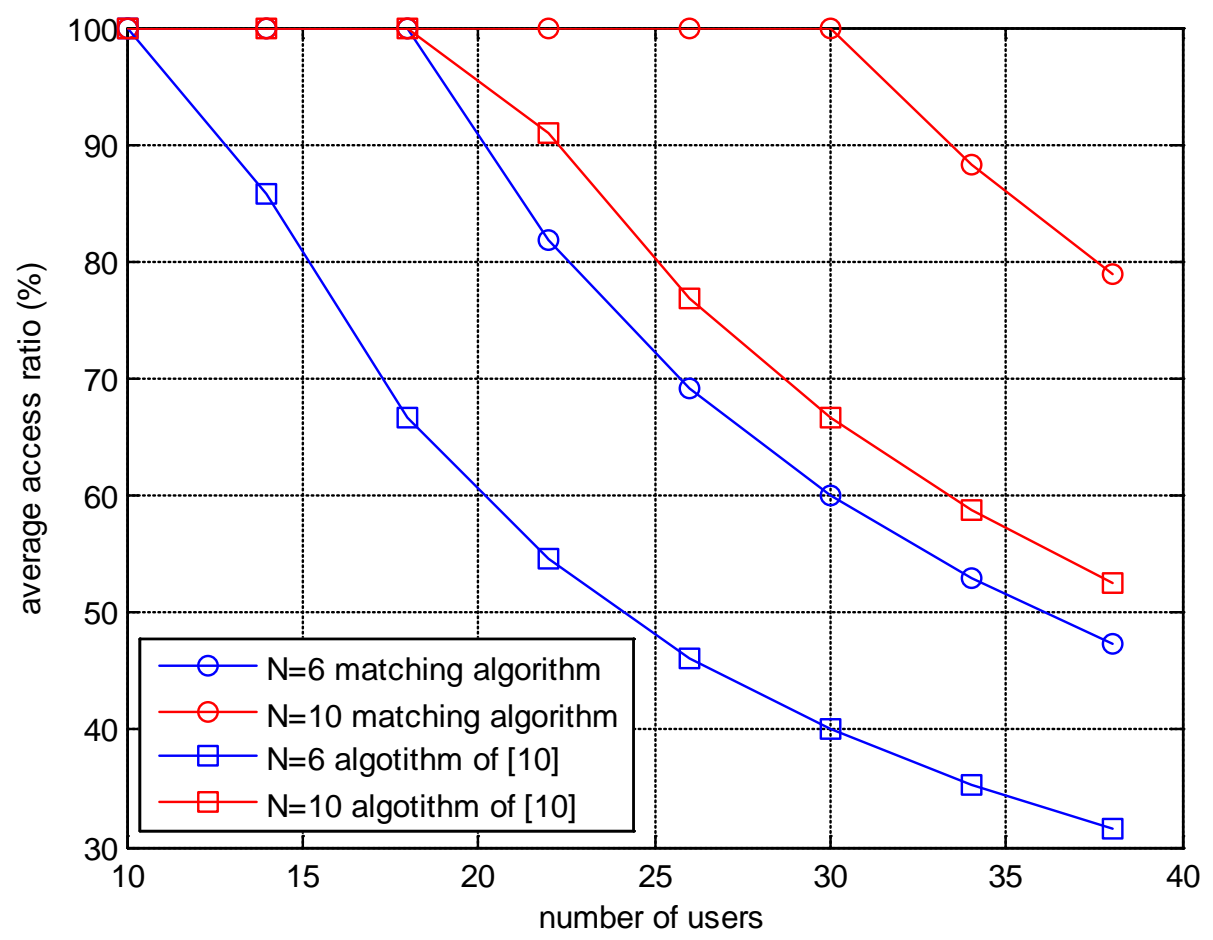

Fig. 4. Comparison on average user access ratio

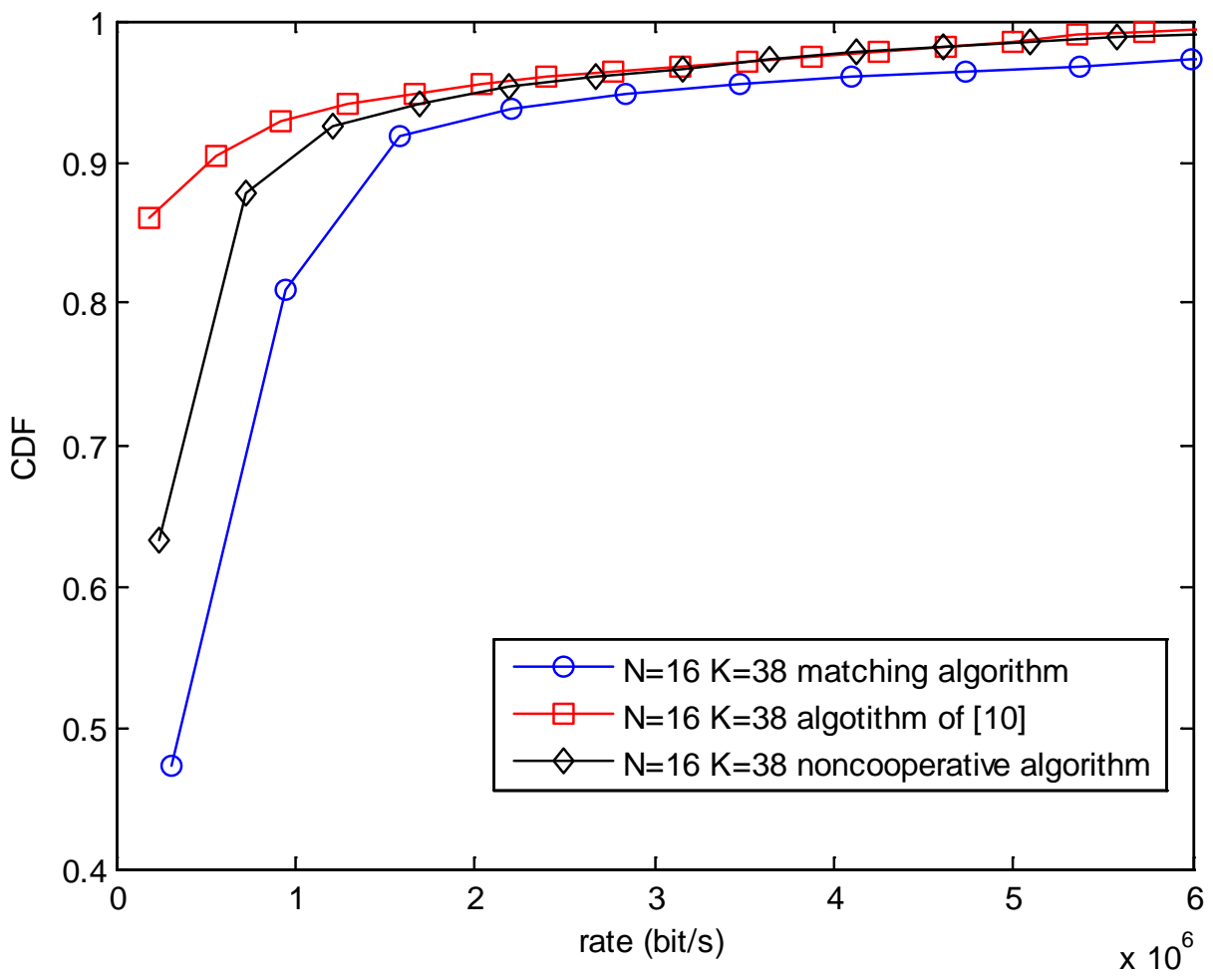

Fig. 5. Comparison on CDF of user rate 
We investigate the distribution of user rates in Fig. $\mathbf{5}$ when the number of users is 38 and number of small cells is 16 . This figure compares the CDF of user rate of three algorithms, where the non-cooperative algorithm allows users to access small cells using non-cooperative game with rate as its utility. It shows that our algorithm performs better on rate than other algorithms, for our algorithm considers the externality and finds the optimal matching, while in [10] a small cell only serves two users and non-cooperative algorithm is not optimal.

\section{Conclusion}

In this paper, the promising technologies of NOMA and SIC are introduced to serve multi users simultaneously in the same channel of an energy harvesting small cell, and recover information from an overlapped signal. To address the user access problem, we model this issue as a rate based two-sided many-to-one matching game with its utility being rate. Considering the dynamic influence of user small cell association in matching, we also propose a matching game algorithm to solve the externality and prove its stability. The simulation results and analyses prove that our algorithm has advantages regarding system capacity and efficiency compared with others.

\section{References}

[1] F Zhou, Y Wu, Y C Liang, Z Li, Y Wang and K K Wong, "State of the Art, Taxonomy, and Open Issues on Cognitive Radio Networks with NOMA," IEEE Wireless Communications, vol. 25, no. 2, pp. 100-108, April, 2018. Article (CrossRef Link).

[2] F Zhou, Y Wu, R Q Hu and K K Wong, "Energy-Efficient NOMA Enabled Heterogeneous Cloud Radio Access Networks,” IEEE Network, vol. 32, no. 2, pp. 152-160, March, 2018.

Article (CrossRef Link).

[3] F Zhou, Z Chu, H Sun and VCM Leung, "Resource Allocation for Secure MISO-NOMA Cognitive Radios Relying on SWIPT,” 2018. Article (CrossRef Link)

[4] H Gao, W Ejaz and M Jo, "Cooperative Wireless Energy Harvesting and Spectrum Sharing in 5G Networks,” IEEE Access, vol. 4, pp. 3647-3658, June, 2016. Article (CrossRef Link).

[5] QT Vien, TA Le, CV Phan and M O Agyeman, "An energy-efficient NOMA for small cells in heterogeneous CRAN under QoS," in Proc. of European Wireless 2017; 23th European Wireless Conference. VDE, pp.80-85, May 17-19, 2017. Article (CrossRef Link)

[6] J A Oviedo and H R Sadjadpour, "A Fair Power Allocation Approach to NOMA in Multi-user SISO Systems," IEEE Transactions on Vehicular Technology, vol. 66, no. 9, pp. 7974-7985, Sept, 2017. Article (CrossRef Link).

[7] J Zhu, J Wang, Y Huang, S He, X You and L Yang, "On Optimal Power Allocation for Downlink Non-Orthogonal Multiple Access Systems," IEEE Journal on Selected Areas in Communications, vol. 35, no. 15, PP. 2744-2757, Dec, 2017. Article (CrossRef Link).

[8] T Sanguanpuak, S Guruacharya, N Rajatheva, M Bennis and M Latva-Aho, "Multi-Operator Spectrum Sharing for Small Cell Networks: A Matching Game Perspective,” IEEE Transactions on Wireless Communications, vol. 16, no. 6, pp.3761-3774, June, 2017. Article (CrossRef Link).

[9] J Zhao, Y Liu, K K Chai, A Nallanathan, Y Chen and Z Han, "Spectrum Allocation and Power Control for Non-Orthogonal Multiple Access in HetNets," IEEE Transactions on Wireless Communications, vol. 16, no. 9, pp. 5825-5837, Sept, 2017. Article (CrossRef Link).

[10] W Liang, Z Ding, Y Li and L Song. "User Pairing for Downlink Non-Orthogonal Multiple Access Networks using Matching Algorithm,” IEEE Transactions on Communications, vol. 65, no. 16, pp. 5319-5332, Dec, 2017. Article (CrossRef Link). 
[11] B Di, L Song and Y Li, “Sub-Channel Assignment, Power Allocation, and User Scheduling for Non-Orthogonal Multiple Access Networks,” IEEE Transactions on Wireless Communications, vol. 16, no. 11, pp. 7686-7698, Nov, 2016. Article (CrossRef Link).

[12] M B Shahab, M F Kader and S Y Shin, "A Virtual User Pairing Scheme to Optimally Utilize the Spectrum of Unpaired Users in Non-orthogonal Multiple Access,” IEEE Signal Processing Letters, vol. 23, no.12, pp. 1766-1770, Dec, 2016. Article (CrossRef Link).

[13] F Zhou, Z Chu, H Sun, RQ Hu and L Hanzo, "Artificial Noise Aided Secure Cognitive Beamforming for Cooperative MISO-NOMA Using SWIPT,” IEEE Journal on Selected Areas in Communications, PP(99):1-1, 2018. Article (CrossRef Link).

[14] W Li, W Zheng, X Wen and T Su, "Dynamic Clustering Based Sub-Band Allocation in Dense Femtocell Environments,” in Proc. of Vehicular Technology Conference. IEEE, pp. 1-5, May 6-9, 2012. Article (CrossRef Link).

[15] D Liu, Y Xu, L Shen and Y Xu, "Self-organising multiuser matching in cellular networks: a score-based mutually beneficial approach,” Iet Communications, vol. 10, no. 15, pp. 1928-1937, Oct, 2016. Article (CrossRef Link).

[16] D Liu, Y Xu, Y Xu, C Ding, K Xu and Y Xu, "Distributed satisfaction-aware relay assignment: a novel matching-game approach,” Transactions on Emerging Telecommunications Technologies, vol. 27, no. 8, pp. 1087-1096, August, 2016. Article (CrossRef Link).

[17] Q Han, B Yang, C Chen and X Guan, "Matching-Based Cell Selection for Proportional Fair Throughput Boosting via Dual-Connectivity,” in Proc. of Wireless Communications and NETWORKING Conference. IEEE, pp. 1-6, March 19-22, 2017. Article (CrossRef Link).

[18] J Zhao, Y Liu, K K Chai, Y Chen and M Elkashlan, "Many-to-Many Matching with Externalities for Device-to-Device Communications,” IEEE Wireless Communications Letters, vol. 6, no. 1, pp. 138-141, Feb, 2016. Article (CrossRef Link).

[19] D Zhang, Z Chen, M K Awad, N Zhang, H Zhou and X S Shen, "Utility-Optimal Resource Management and Allocation Algorithm for Energy Harvesting Cognitive Radio Sensor Networks,” IEEE Journal on Selected Areas in Communications, vol. 34, no. 12, pp. 3552-3565, Dec, 2016. Article (CrossRef Link).

[20] H Claussen, LTW Ho and L G Samuel, "Self-optimization of coverage for femtocell deployments,” Wireless Telecommunications Symposium. IEEE, pp. 278-285, April 24-26, 2008. Article (CrossRef Link).

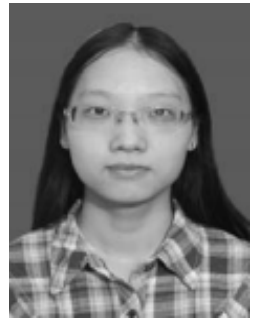

Xueting Wang was born in Anhui Province, China, in 1994. She received the B.E. degree in Communication Engineering from Nanjing University of Posts and Telecommunications, Nanjing, in 2016. She is now pursuing master's degree in the Department of Telecommunication and Information Engineering, Nanjing University of Posts and Telecommunications. Her research interests include resource allocation in energy-harvesting small cell networks, mobile communication and wireless technology.

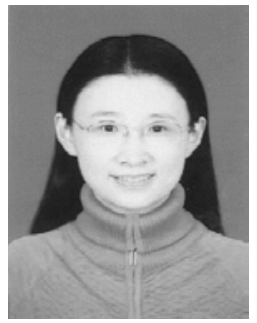

Qi Zhu (corresponding author) was born in Suzhou, Jiangsu, China, in 1965. She received the M.S. degree in radio engineering from Nanjing University of Posts and Telecommunications in 1989. Now she is a professor in the Department of Telecommunication and Information Engineering, Nanjing University of Posts and Telecommunications, Jiangsu, China. Her research interests focus on technology of next generation communication, broadband wireless access, OFDM, channel and source coding, dynamic allocation of radio resources. 